\title{
Diferencias entre hombres y mujeres en los trastornos de ansiedad: una aproximación psicobiológica
}

\author{
M. Carmen Arenas ${ }^{1}$, Araceli Puigcerver ${ }^{2}$ \\ ${ }^{1}$ Dpto. Psicobiología, Facultad de Psicología, Universitat de València, \\ ${ }^{2}$ Dpto. Psicobiología y Metodología de las Ciencias del Comportamiento, Facultad de Psicología, Universidad de Málaga
}

Disponible online 22 diciembre de 2009

\begin{abstract}
Los trastornos de ansiedad son, en la actualidad, enfermedades psiquiátricas muy frecuentes e incapacitantes. Las mujeres en edad reproductiva son más vulnerables a desarrollar trastornos de ansiedad, aproximadamente entre 2 a 3 veces más que los hombres. Ser hombre o mujer no sólo puede influir en la prevalencia de los trastornos mentales, sino también en la manifestación y expresión de los síntomas, la voluntad para solicitar asistencia médica o psicológica, el curso de la enfermedad, incluso en la respuesta al tratamiento. Cada vez hay pruebas más sólidas de que existen diferencias entre ambos sexos respecto a la anatomía cerebral, la neuroquímica y los patrones de activación y respuesta a los estímulos ambientales; diferencias que pueden influir en la etiología y el curso de los trastornos psiquiátricos. Sin embargo, poco se conoce sobre los factores de riesgo que inducen a las mujeres a desarrollar ciertas psicopatologías. Por ello, en este trabajo pretendemos presentar una breve revisión sobre los aspectos psicobiológicos que pueden contribuir a las diferencias de sexo en los trastornos de ansiedad.
\end{abstract}

Palabras clave: trastornos de ansiedad, diferencias sexuales

Anxiety disorders are common in both men and women and are particularly disabling for the sufferer. Women of reproductive age are more vulnerable to developing these mental disorders than men; in fact their prevalence is 2-3 times higher among females than among males. Sex differences have also been reported in relation to the manifestation and expression of symptoms, the will to request medical or psychological assistance, the course of the disease, and even in the response to treatment. These sex differences may be attributable to multiple factors, such as genetic predisposition, anatomy, hormones and environment. However, very little is known about the risk factors for women with respect to developing anxiety disorders, and so the origins of sex differences in these disorders is an important topic of research. We consider that variations in stress reactivity may be one of the mechanisms underlying gender differences in anxiety disorders. The purpose of this brief review is to highlight data on the psychobiological factors that make women more prone to suffering anxiety disorders than men.

Keywords: anxiety disorders, sex differences

Correspondencia: Dra. M. Carmen Arenas. Dpto. Psicobiología, Facultad de Psicología, Universitat de Vàlencia, Blasco Ibañez, 21, 46010, Valencia. E-mail: carmen.arenas@uv.es 
Los trastornos de ansiedad y los del estado de ánimo son una de las enfermedades mentales más frecuentes en la actualidad. Sólo en Estados Unidos se estima que 1 de cada 5 personas sufrirá en su vida uno o varios de estos trastornos (Kessler et al., 1994). Según los resultados del proyecto Estudio Europeo de la Epidemiología de los Trastornos Mentales (European Study of the Epidemiology of Mental Disorders, ESEMeD), el 13,6\% de la población de seis países europeos (Bélgica, Francia, Alemania, Italia, Holanda y España) ha padecido algún trastorno de ansiedad a lo largo de su vida (prevalencia-vida) y el 6,4\% en el último año del estudio (prevalencia-año) (Alonso et al., 2004).

Las mujeres en edad reproductiva son más vulnerables a desarrollar trastornos de ansiedad que los hombres, aproximadamente entre 2 a 3 veces más. De hecho, el 17,5\% de las mujeres frente al 9,5\% de los hombres habían sufrido algún trastorno de ansiedad a lo largo de su vida; mientras que el $8,7 \%$ de las mujeres frente al 3,8\% de los hombres habían padecido un trastorno de ansiedad en el último año (Alonso et al., 2004; CarrascoGalán y Espinar-Fellmann, 2008). Ser hombre o mujer no sólo puede influir en la prevalencia de los trastornos mentales, sino también en la manifestación y expresión de los síntomas, la voluntad para solicitar asistencia médica o psicológica, el curso de la enfermedad, incluso en la respuesta al tratamiento (Phillips y First, 2009; Wisner y Dolan-Sewell, 2009).

El estudio de las diferencias de género en los trastornos mentales es un campo de investigación que recientemente está en alza a nivel preclínico (Horst et al., 2009). Una búsqueda en el MedLine utilizando los términos sexo (sex), género (gender) $\mathrm{y}$ ansiedad (anxiety) nos revela que el número de trabajos con seres humanos publicados en inglés en 1990 fue de 96, frente a los 664 en 2006 (Wisner y Dolan-Sewell, 2009). Sin embargo, todavía hace falta mucha investigación para aclarar la relación entre género y psicopatología, como vienen denunciando instituciones internacionales de la salud como American Psychological Association, American Psychiatric Association, National Institute of Mental Health y la Organización Mundial de la Salud (OMS) (Phillips y First, 2009; Wisner y Dolan-Sewell, 2009). La OMS considera que no se estaba prestado apenas atención a los determinantes específicos de cada sexo en los mecanismos que promueven y protegen la salud mental y fomentan la resistencia al estrés y a la adversidad (OMS, 2000). Dos de los objetivos principales que se propuso esta institución al respecto, fueron recoger datos de la prevalencia y las causas de los problemas de salud mental en las mujeres, así como de los factores mediadores y protectores, para formular y poner en funcionamiento medidas de salud que atiendan las necesidades y preocupaciones concretas de las mujeres desde la infancia hasta la vejez (OMS, 2001).

Se han identificado un conjunto de factores que pueden explicar el predominio femenino en los trastornos de ansiedad, así como las diferencias entre los sexos en otros trastornos psiquiátricos. Un grupo de estos factores serían los psicosociales y socioculturales que incluyen diferencias en el tipo de afron- tamiento de la persona, rol sexual, pobreza, nivel educativo, estado civil, nivel de ingresos, apoyo social, aislamiento social, adversidades durante la infancia, cambios sociales, normas culturales y vulnerabilidad a la exposición y reactividad ante acontecimientos vitales estresantes. Otros factores que sugieren diferencias de sexo son la comorbilidad previa, la predisposición genética, los rasgos de la personalidad, las hormonas sexuales, la reactividad endocrina frente al estrés, los sistemas de neurotransmisión y los determinantes neuropsicológicos (Grant y Weissman, 2009). Hay consenso en considerar que los factores psicosociales y socioculturales no pueden explicar todas las diferencias observadas entre ambos sexos, y dada la coherencia de los hallazgos en los distintos grupos culturales, la conclusión es que las diferencias de género en las tasas de los trastornos psiquiátricos son en gran parte psicobiológicas. Evaluar cuales de los factores explicativos de las diferencias entre los sexos son de naturaleza biológica y cuales de naturaleza social, nos permitirá conocer cuales son inmutables y cuales no (Dalla y Shors, 2009).

A pesar de la escasez de estudios, cada vez hay más pruebas sólidas de que existen diferencias entre ambos sexos respecto a la anatomía cerebral, la neuroquímica y los patrones de activación y respuesta a los estímulos ambientales, así como diferencias respecto a la fisiología y fisiopatología de otros sistemas corporales, diferencias que pueden influir en la etiología y el curso de los trastornos psiquiátricos (Altemus, 2009). Por ello, en este trabajo pretendemos presentar una panorámica general de la investigación que se está llevando a cabo sobre los aspectos psicobiológicos que pueden contribuir a las diferencias de sexo en la prevalencia, los patrones sintomáticos y las respuestas terapéuticas centrándonos en el contexto de los trastornos de ansiedad.

Diferencias sexuales en los trastornos de ansiedad

La ansiedad puede ser conceptualizada como un estado negativo con tres componentes, uno fisiológico, la hipervigilancia, otro afectivo, el miedo, y otro cognitivo, la indefensión; y cuya principal característica es la sensación de incontrolabilidad sobre posibles amenazas o daños (Chua y Dolan, 2000). Aunque la ansiedad es una reacción psicológica normal que tiene la función de prepararnos para peligros futuros, también representa el síntoma psiquiátrico más común. Todos los trastornos de ansiedad se caracterizan por presentar síntomas de ansiedad, pero son muy distintas las características de cada trastorno. El DSM-IVTR (Diganostic and Statistical Manual of Mental Disorders) distingue entre trastorno de angustia (con agorafobia o sin ella) o agorafobia sin trastorno de angustia, trastorno de ansiedad generalizada, trastorno obsesivo-compulsivo, fobia específica, fobia social, trastorno por estrés postraumático, trastorno por estrés agudo, trastorno de ansiedad debido a enfermedad médica, trastorno de ansiedad inducido por sustancias y trastorno de ansiedad no especificado (American Psychiatric Association, 2000). 
El trastorno de angustia se define como una alteración psiquiátrica caracterizada por ataques de pánico inesperados y repetidos. Los ataques de pánico son limitados en el tiempo, altamente aversivos y están acompañados de un amplio rango de consecuencias fisiológicas y psicológicas negativas. Los estudios epidemiológicos y clínicos han corroborado que el trastorno de angustia es doblemente frecuente en las mujeres y que su edad de inicio es diferente en cada sexo; en hombres se presenta entre los 15 y los 24 años mientras que en las mujeres se inicia algo más tarde, entre los 35 y los 44 años (Eaton et al., 1994). El trastorno de angustia en mujeres tiende a ser más severo que en los hombres y se asocia con tasas elevadas de comorbilidad con agorafobia, trastorno de ansiedad generalizada y trastorno de somatización. También encontramos diferencias sexuales en su sintomatología, ya que en las mujeres el pánico presenta predominantemente síntomas respiratorios, mientras que en los hombres son síntomas gastrointestinales acompañados de sudoración intensa (Leskin y Sheikh, 2004).

Muy recientemente, desde el campo de la neuroimagen, se ha podido comprobar cambios sexo-dimórficos en distintas estructuras cerebrales de pacientes con trastorno de angustia. Los hombres presentaron una mayor reducción en la amígdala derecha y en la corteza insular bilateral, mientras que las mujeres mostraron una disminución más marcada en el giro temporal derecho, en la corteza prefrontal dorsolateral y ventrolateral, en la corteza parietal y en el tálamo (Asami et al., 2009). Estos hallazgos confirmarían la implicación de la corteza prefrontal y de la amígdala en la patofisiología de este trastorno y ofrecerían una posible explicación de las diferencias sexuales en su sintomatología.

Las fluctuaciones hormonales se han asociado tradicionalmente con un aumento en la incidencia del trastorno de angustia en mujeres. Sin embargo, aunque no se conocen las interrelaciones concretas entre la ciclicidad menstrual y el trastorno de angustia, sí se ha verificado que los cambios en las hormonas premenstruales contribuyen a los episodios de pánico. De hecho, a pesar de que el empeoramiento premenstrual del pánico no se ha replicado en todos los estudios, sí se ha observado que las mujeres con trastorno premenstrual (TPM) tienden a presentar pánico tras la exposición a distintos agentes panicogénicos como el lactato, el $\mathrm{CO}_{2}$, o los antagonistas de la colecistoquinina (Le Melledo et al, 2000). Se conoce que los metabolitos de la progesterona pueden tener efectos ansiolíticos debido a su acción agonista sobre los receptores GABA/ BZD (Seeman, 1997), por lo que una posible alteración en este complejo receptor podría explicar los factores que vinculan las respuestas de pánico, el ciclo reproductivo femenino y el TPM (Leskin y Sheik, 2004).

Otro posible mecanismo explicativo de las diferencias de sexo en la patofisiología del pánico podrían ser las diferencias descritas en la sensibilidad del receptor $\beta$-adrenérgico entre hombres y mujeres. La norepinefrina se relaciona con la etio- logía y sintomatología de los ataques de pánico, presentando los pacientes una respuesta anormal tras la estimulación de los receptores $\beta$-adrenérgicos. Concretamente las mujeres con trastorno de angustia presentaron una reducción en la sensibilidad del receptor que no fue observada en los hombres (Kim et al., 2004).

La agorafobia es una de las consecuencias más discapacitante del trastorno de angustia. Consiste en tener miedo a presentar ataques de pánico, lo que lleva a los sujetos a confinarse en el hogar, un espacio donde pueden controlar las actividades y los estímulos. Afecta a un 3-4\% de hombres frente a un 7-9\% de mujeres, presentando éstas mayor severidad en los síntomas y mayor deterioro de la calidad de vida (Altemus y Epstein, 2008; Kessler et al., 1994).

El trastorno de ansiedad generalizada (TAG) consiste en una preocupación excesiva e incontrolable sobre múltiples problemas cotidianos. Entre los síntomas se incluyen la tensión muscular, la fatiga, el insomnio, la inquietud, la falta de concentración y la irritabilidad. La tasa prevalencia-vida es del 4\% en los hombres, frente al 7\% en las mujeres (Altemus y Epstein, 2008; Kessler et al., 1994). Esta divergencia entre los sexos comienza a una edad temprana y continúa en la adolescencia y en la edad adulta (McLean y Anderson, 2009). Son numerosos los estudios que han verificado un empeoramiento premenstrual de los síntomas del TAG (Peer et al., 2008), aunque se desconocen los mecanismos por los que algunas mujeres presentan este agravamiento del trastorno. Sin embargo, a diferencia del trastorno de angustia, el curso clínico del TAG suele ser el mismo en hombres y en mujeres, así como el riesgo de remisión y de recaídas (Yonkers y Kidner, 2002).

El trastorno obsesivo compulsivo (TOC) es una enfermedad severa y frecuente que afecta del 1 al 3\% de la población (Weissman et al., 1994). Una persona con TOC sufre pensamientos obsesivos y repetitivos y tiene muchos miedos (a contaminarse, a herir a otros o a sí mismo, dudas morales,...) que le inducen a realizar compulsivamente conductas ritualizadas que le disminuyen la ansiedad. El TOC es el único trastorno de ansiedad que no presenta diferencias sexuales en su incidencia, a pesar de la creencia general de que el trastorno es más frecuente en los hombres (Lochner et al., 2004). No obstante, existen diferencias de sexo en la expresión y en la edad de inicio de los síntomas del trastorno, en el curso y en la respuesta al tratamiento del mismo (Torresan et al., 2009; Yonkers y Kidler, 2002). El comienzo de los primeros síntomas es más precoz en los hombres, coincidiendo con la pubertad o la primera juventud, mientras que en la mujer no suele manifestarse antes de los 20 años, normalmente asociado a embarazos (Boggetto et al., 1999). Con respecto a los síntomas, las mujeres muestran más compulsiones dañinas sobre limpieza y comprobaciones, mientras que los hombres presentan más tics, mayor gravedad en los síntomas en general y un peor pronóstico (Lochner et al., 2004).

Algunas investigaciones sobre el TOC se han centrado en las posibles influencias hormonales. Se ha observado cambios 
concretos en los síntomas de mujeres con TOC tanto en la fase premenstrual/menstrual, como tras el embarazo y la menopausia, confirmándose la interrelación entre los cambios cíclicos menstruales/reproductivos y las recaídas y las fluctuaciones en los síntomas de este trastorno (Lochner et al., 2004).

Como muchos trastornos psiquiátricos, el TOC tiene una predisposición genética subyacente en muchos de los pacientes. Uno de los parámetros genéticos mejor estudiados en el TOC es la expresión genética de la catecol-O-metil-transferasa (COMT). Parece que la regulación a la baja de la COMT es un factor que contribuye a la predisposición al TOC sólo en pacientes varones. Sin embargo, carecemos de una explicación completa y no se dispone de resultados concluyentes (Pooley et al., 2007; Wang et al., 2009). Aunque los datos genéticos son prometedores, de momento es prematura una conclusión sólida salvo la demostración de un patrón sexo-dimórfico en la susceptibilidad genética a presentar el TOC. Y a pesar de los avances experimentados en los últimos 10 años, se sabe aún muy poco acerca de la etiopatogénesis del trastorno, faltando todavía mucha información sobre el por qué de las diferencias de género.

Una fobia es un miedo excesivo a un objeto o situación particular. Entre las fobias específicas más comunes están las fobias a animales, con una prevalencia-vida del 5\% en los hombres y del $11 \%$ en las mujeres; las fobias a situaciones como volar, sitios cerrados, o altos, con una prevalencia-vida del $9 \%$ en hombres y un $12 \%$ en mujeres; y la fobia social, que se presenta en un $11 \%$ de los hombres frente al $16 \%$ de las mujeres (Altemus y Epstein, 2008; Angst, 1997; Kessler et al., 1994). Al igual que en el TAG, no se han observado diferencias de sexo en el curso clínico de estos trastornos. A pesar de que no se dispone todavía de información acerca del impacto de las hormonas reproductivas femeninas en el curso y severidad de la ansiedad social, no se duda de la influencia de estas hormonas tanto en la conducta de evitación en las mujeres como en la ansiedad social. Se ha observado que las mujeres diagnosticadas suelen presentar un empeoramiento de los síntomas en la fase premenstrual del ciclo, al igual que las mujeres embarazadas exhiben un aumento en los niveles de ansiedad social en el primer trimestre del embarazo (Peer et al., 2008).

El trastorno por estrés postraumático (Posttraumatic Stress Disorder, PTSD) sobreviene como consecuencia de la exposición a un evento traumático, psicológico o físico, que provoca un estrés agudo o crónico. Se caracteriza por la reexperimentación del trauma a través de sueños o flashbacks, por la hipervigilancia y por la evitación de pensamientos o estímulos que puedan recordar la situación traumática (Yehuda y LeDoux, 2007). Como la mayoría de los trastornos de ansiedad, son las mujeres quienes manifiestan en mayor proporción el PTSD, con una prevalencia-vida del 10\% en las mujeres frente al 5\% de los hombres (Altemus y Epstein, 2008; Kessler et al., 1995; Olff et al., 2007).
Una de las explicaciones a las diferencias de sexo en la prevalencia del PTSD es la posibilidad de que las mujeres se expongan en mayor medida a situaciones traumáticas que los hombres. Sin embargo, un exhaustivo estudio de Tolin y Foa (2006) ha demostrado que, en general, son los hombres los que se exponen con mayor frecuencia a eventos amenazantes o traumáticos (accidentes, ataques no sexuales, combate o guerra, fuego o desastres,...), estando las mujeres más expuestas sólo en el caso de abusos y ataques sexuales. Además, siguen apareciendo diferencias de sexo en el desarrollo de este trastorno a favor de la mujer, aún cuando se controla la varianza atribuible a los abusos sexuales o incluso cuando se examina la frecuencia y severidad del PTSD entre hombres y mujeres expuestos a un mismo evento traumático. Por tanto, la mayor prevalencia en las mujeres no puede explicarse por el mayor riesgo de sufrir un abuso o ataque sexual (Olff et al., 2007; Tolin y Foa, 2006).

La influencia sobre la respuesta neuroendocrina al estrés de los patrones específicos de cada sexo en la evaluación cognitiva y en los procesos de afrontamiento de la situación traumática ha sido considerada como posible causa del mayor riesgo de las mujeres a desarrollar PTSD (Olff et al., 2007). La respuesta particular a amenazas extremas, pasa de ser adaptativa a desadaptativa en función de numerosos factores; y es el proceso de evaluación del evento estresante el primer paso en la cascada de respuestas psicobiológicas. Se ha sugerido que el que las mujeres perciban el evento de forma más amenazante y con mayor pérdida de control que los hombres, puede contribuir a la mayor prevalencia del PTSD en ellas, por provocar diferencias en la respuesta del eje hipotalámico-pituitario-adrenal (HPA). Existen evidencias de que la conducta de afrontamiento del estrés y las respuestas fisiológicas relacionadas son diferentes en roedores machos y hembras, lo cual se atribuye a diferencias anatómicas, influencias hormonales y factores ambientales. Se ha demostrado que, usando un paradigma de estrés crónico, los machos adoptan la respuesta típica de lucha o huída ("fight or flight”), mientras que las hembras mostrarían una respuesta más defensiva y pasiva, es decir, lo que se ha denominado una conducta más protectora o amistosa ("tend and befriend") (Taylor et al., 2000); esto vendría a explicar que las hembras alojadas en grupo presenten significativamente menos efectos negativos por la exposición al estrés que las hembras alojadas individualmente, no habiéndose encontrado estas correlaciones en los machos (Horst et al., 2009). Estos datos preclínicos corroboran los estudios epidemiológicos en seres humanos que muestran un efecto protector del apoyo social sobre el riesgo a desarrollar trastornos relacionados con el estrés en las mujeres (Kendler et al., 2005).

Las principales alteraciones neuroendocrinas observadas en los sujetos con PTSD son un incremento de la activación simpática y de la hormona liberadora de la corticotropina (CRH), con una reducción del cortisol debido a una hipersensibilidad del feedback negativo en el eje HPA (Yehuda y LeDoux, 2007). Se ha observado en pacientes con una hipocortisolémia diag- 
nosticados de PTSD tras sufrir un accidente de coche, que los hombres tenían niveles más altos de cortisol en saliva que las mujeres, presentando una variación diurna atípica diferente: mientras los hombres tendían a aumentar sus niveles, las mujeres mostraron una reducción de la concentración de cortisol a lo largo del día (Paris et al., in press). Actualmente, se considera que los niveles bajos de cortisol en orina son un predictor del grado de PTSD que presenta el sujeto, y que las alteraciones relacionadas con el cortisol en este trastorno reflejarían unos factores de vulnerabilidad preexistente al trauma (Yehuda y LeDoux, 2007; Paris et al., in press).

Otra posible explicación de las diferencias de sexo en la incidencia del PTSD podría ser el hecho de que las mujeres muestran en mayor medida la disociación peritraumática (DPT) en comparación con los hombres. La DPT es un estado de conciencia limitada o distorsionada durante el tiempo que dura el evento traumático o inmediatamente después. Se ha vinculado a una actividad reducida del eje HPA y a las respuestas defensivas y pasivas en situaciones estresantes, por lo que se ha asociado también al desarrollo de PTSD y se considera un posible predictor de este trastorno (Olff et al., 2007).

Algunos estudios han mostrado que los sujetos que presentan una mayor activación del sistema nervioso simpático durante la fase aguda tras el trauma, son los que posteriormente desarrollan el PTSD. Y aunque los niveles elevados de cortisol son más comunes en hombres que en mujeres supervivientes de una catástrofe natural, los datos disponibles también muestran una mayor presencia de síntomas fisiológicos agudos, especialmente hiperactivación, en las mujeres que en los hombres después de sufrir un desastre natural (Olff et al., 2007). Se ha sugerido que esa hiperactivación tras un estrés extremo puede dar lugar al desarrollo de "sobreconsolidación" de memorias y, así, contribuir al desarrollo del PTSD (Paris et al., in press).

Otro aspecto a considerar para entender las diferencias de sexo en la vulnerabilidad al PTSD, es el hecho de que las mujeres presentan una recuperación más lenta que los hombres en la respuesta hormonal al estrés. Se sabe que una rápida recuperación de la respuesta de estrés es importante para superar el trauma, y niveles más altos de oxitocina se han asociado a un restablecimiento del eje HPA más rápida en mujeres que en hombres después de un estrés agudo en el laboratorio (Olf et al., 2007).

Por último, indicar que existen datos que relacionan el PTSD con cambios en el sistema inmunitario que podrían justificar, en parte, las diferencias de sexo en este trastorno. Se ha descrito un incremento en la activación de los linfocitos CD45 (células de memoria del sistema inmunológico) mayor en pacientes diagnosticados con PTSD que en los controles (Wilson et al., 1999), alteraciones semejantes a las de patologías autoinmunes como el lupus eritematoso sistémico. Es conocido que las mujeres presentan una respuesta inmunitaria más vigorosa que los hombres, pero también una mayor incidencia de enfermedades autoinmunes, como el lupus eritematoso sistémico (entre 7-10 veces más), la artritis reumatoide o la esclerosis múltiple (entre 2-3 veces más) (Darnall y Suarez, 2009).

Factores psicobiológicos explicativos de las diferencias sexuales en los trastornos de ansiedad

Durante muchos años, las diferencias entre los sexos y el papel que las hormonas gonadales tenían en estas diferencias, se estudiaron exclusivamente centrándose en la conducta sexual. Actualmente, al margen de controversias, se acepta que la diferenciación sexual implica diversas variantes estructurales, químicas y funcionales entre el cerebro del varón y el de la mujer, que afectan a todos los ámbitos del sujeto (Cahill, 2006; Cahill, 2009).

Es bien conocido que la diferenciación sexual está influida por factores genéticos y hormonales, y que este proceso se lleva a cabo en varias fases distintas. Primero se establece el sexo genético o cromosómico (XX vs. XY), después el sexo gonadal (ovarios vs. testículos), posteriormente se desarrolla el fenotipo genital y, finalmente en la pubertad, se establecen los caracteres sexuales secundarios. Aunque las hormonas sexuales son elementos clave para el desarrollo de algunos de estos procesos, no hay que olvidar que el papel de estas hormonas perdura durante toda la vida del individuo, contribuyendo así, día a día, a su diferenciación sexual.

Los estudios de familias han demostrado repetidas veces que los factores genéticos juegan un papel importante, aunque no determinante, en la aparición de los trastornos de ansiedad. No obstante, las investigaciones genéticas no han presentado, hasta ahora, ningún gen o genes como candidatos que predispongan a desarrollar estos trastornos. Un posible mecanismo implicado son los alelos potencialmente vulnerables a desarrollar ansiedad, siendo uno de ellos el alelo Ser23 para el receptor 5HT2C. Los agonistas de este receptor serotoninérgico tienen efectos ansiógenos en sujetos voluntarios e incrementan los síntomas de la ansiedad en sujetos con TOC o con trastorno de angustia. Pero los resultados hasta el momento son contradictorios, pues mientras algún estudio ha encontrado que este alelo se encuentra con mayor frecuencia en mujeres que en hombres, otros trabajos han mostrado la proporción contraria (Altemus y Epstein, 2008).

Por lo que respecta a la neuroanatomía, la amígdala y el hipocampo están muy implicadas en las emociones y en la respuesta de estrés. En los últimos años, se ha demostrado que estas áreas cerebrales son sexualmente dimórficas en su estructura; mientras que la amígdala es mayor en los hombres que en las mujeres, el hipocampo es mayor en las mujeres que en los hombres. Se ha comprobado además, que la amígdala y el hipocampo reaccionan al ambiente de forma diferente en cada sexo. Cuando se evaluó la actividad de la amígdala, crucial para el recuerdo de sucesos emotivos, de sujetos que contemplaban diapositivas con imágenes perturbadoras, los hombres que manifestaron una respuesta emocional intensa, exhibieron 
mayor actividad en la amígdala derecha, mientras que las mujeres la mostraron en la amígdala izquierda (Cahill et al., 2001, Cahill, 2003). Se ha sugerido que estas diferencias en actividad hemisférica entre los sexos, serían responsables de que las mujeres recuerden más vivamente los detalles de un acontecimiento emotivo y de que los hombres, por el contrario, conserven un recuerdo más global del acontecimiento. En estudios con animales se ha observado que las situaciones de estrés a una edad temprana provocan también consecuencias diferentes en el cerebro de cada sexo. Mientras que la ansiedad por separación de la madre aumentaba la concentración de receptores de serotonina en la amígdala de los machos, la misma situación disminuía la concentración de tales receptores en las hembras. Si ha esto añadimos que la producción de serotonina es un 52\% más elevada en el varón que en la mujer (Cahill, 2006; Cahill, 2009), resulta una línea de investigación muy interesante y prometedora para comprender la predisposición de las mujeres a desarrollar trastornos de ansiedad y del estado de ánimo.

Los estudios epidemiológicos nos muestran que las diferencias entre los sexos a desarrollar trastornos de ansiedad, surgen en la adolescencia y tienden a desaparecer en la menopausia de la mujer. Asimismo, la incidencia de estos trastornos varía según las distintas fases del ciclo hormonal en la que se encuentre la mujer. Si además tenemos en cuenta que los cambios introducidos por las hormonas reproductivas -durante la fase intrauterina y la pubertad, el ciclo menstrual, el embarazo y la menopausia-, modifican claramente la estructura y la función cerebral, todo ello sugiere que las hormonas sexuales juegan un papel determinante en la etiología y en las manifestaciones clínicas de estas patologías (Altemus, 2009; Horst et al., 2009).

Concretamente, que la ansiedad disminuya durante el embarazo y la fase luteínica del ciclo menstrual de la mujer, y que se observe un agravamiento de los síntomas de muchos trastornos de ansiedad tras el parto, parece indicar que la progesterona puede tener un papel importante en las diferencias sexuales de los trastornos de ansiedad. Altemus y Epstein (2008) han planteado una hipótesis interesante para entender la mayor vulnerabilidad de la mujer de nuestra época a desarrollar trastornos de ansiedad. Hasta el siglo pasado, hace poco tiempo evolutivamente hablando, las mujeres permanecían muchos años de su vida adulta, o embarazadas o en periodo de lactancia, lo cual equivalía a que su organismo estuviera mucho tiempo expuesto a niveles elevados de progesterona. Esta hormona y sus metabolitos incrementan la sensibilidad del receptor GABA-A, elemento clave para reducir la respuesta de ansiedad en el cerebro. Lo que plantean estas autoras es que, tal vez, el cambio en las mujeres actuales ha sido demasiado rápido, provocando que su organismo, debido a los niveles más bajos de progesterona que los que eran normales en ellas, presente una mayor susceptibilidad a desarrollar respuestas desproporcionadas y patológicas de ansiedad.

Hay una cantidad importante y creciente de bibliografía en la que se destacan los efectos moduladores de las hormo- nas sexuales, principalmente el estradiol, sobre numerosos sistemas neuroquímicos, tales como el GABA en los núcleos amigdalinos y los sistemas dopaminérgico, serotoninérgico, adrenérgico y colinérgico del prosencéfalo, y sobre los comportamientos asociados a estos neurotransmisores (Horst et al., 2009; McEwen y Alves, 1999; Solomon y Herman, 2009). Se ha propuesto la posibilidad de que las diferencias sexuales en los trastornos psicopatológicos puedan estar relacionadas con las diferencias entre sexos en los sistemas de neurotransmisores y neuropéptidos, destacando, entre otros, los opiáceos, la oxitocina, la vasopresina y la hormona liberadora de la corticotropina (CRH). Aunque los estudios con animales de experimentación han demostrado que muchos de estos sistemas están sustancialmente modulados por los esteroides sexuales y presentan dimorfismo sexual, todavía hay pocos estudios con seres humanos que lo corroboren (Altemus, 2009). No obstante, también, es cierto, que los efectos de las hormonas sexuales no explicarían todas las diferencias entre los sexos, pues se ha observado que no se anulan todas las diferencias con la castración de los machos o la ovariectomía de las hembras.

Como hemos visto, los trastornos de ansiedad suelen estar asociados a situaciones estresante o amenazantes y están, por tanto, muy influenciados por las hormonas del eje HPA (Paris et al., in press). Investigar la extensión de esta influencia, es crítica para entender y prevenir los riesgos asociados al estrés en estos trastornos.

Numerosos estudios han mostrado que la activación del eje HPA en respuesta al estrés es un rasgo sexualmente divergente, tanto en animales experimentales como en seres humanos (Paris et al., in press; Young et al., 2008), sin embargo, los resultados son contradictorios. En estudios preclínicos se ha observado una respuesta del eje HPA más potente en los roedores hembras que en los machos (Young, 1998). Las ratas hembras liberan corticosterona/ACTH más rápido y en mayor cantidad en respuesta a un estresor agudo que los machos (Young et al., 2008). No obstante, los estudios clínicos han indicado que son los hombres quienes presentan una respuesta mayor del eje HPA ante un estrés agudo (Kudielka et al. 2004). Concretamente, las mujeres adultas, entre la pubertad y la menopausia, presentan una respuesta del eje HPA menor que los hombres de la misma edad; y, después de la menopausia y durante la fase luteínica del ciclo menstrual, los niveles de cortisol en respuesta al estrés se incrementan acercándose a los niveles de los hombres (Kudielka y Kirschbaum, 2005).

Determinar esta relación entre sexo y respuesta al estrés puede resultar más difícil cuando se analiza en estudios con seres humanos, dado que la actividad del eje HPA evaluada en sujetos sanos puede cambiar cuando los individuos se encuentran en un estado patológico. La respuesta adaptativa a un estrés agudo se considera que produce un incremento rápido en los niveles de glucocorticoides sin afectar la actividad basal del eje HPA. Estos aumentan el arousal y la energía necesaria para la respuesta conductual de huida-o-lucha (fight-or-flight), además 
de incrementar la consolidación de la memoria de los eventos estresantes, lo cual puede facilitar la respuesta adaptativa en situaciones futuras similares. Por otro lado, las diferencias sexuales en respuesta a un estrés agudo pueden influir en la respuesta a un estrés crónico o implacable, teniendo implicaciones importantes para los estados patológicos asociados. A veces, debido a una sobreactivación del eje HPA, el incremento de los glucocorticoides en respuesta al estrés puede ser mayor y más persistente en el tiempo de lo que debería, o producirse ante un estresor poco robusto. Este estado alterado del eje puede provocar una disminución de los niveles basales de glucocorticoides (como se ha observado en el PTSD), o por el contrario puede incrementar la respuesta del eje (como en los trastornos de pánico y otros trastornos de ansiedad). Los factores que pueden inducir esta alteración no son bien conocidos todavía, aunque es probable que dependan de interacciones complejas entre factores epigenéticos, activacionales y organizacionales. Lo que sí se sabe bien es que el estrés crónico puede alterar una gran variedad de funciones fisiológicas periféricas (como procesos cardiovasculares, hepáticos, inmunológicos y digestivos), además de provocar reducciones centrales en la eficacia neuronal y en la neurogénesis principalmente del hipocampo (McEwen, 2002; McEwen, 2007).

Cada vez disponemos de más pruebas que indican que los animales experimentales del sexo femenino, a pesar de presentar una respuesta más potente del eje HPA al estrés, son más resistentes a muchos de los cambios neurobiológicos y comportamentales que experimentan los individuos del sexo masculino frente al estrés agudo y crónico. Una posibilidad de conciliación entre la evidente resistencia femenina a los efectos neurobiológicos del estrés y la mayor prevalencia de los trastornos de ansiedad en las mujeres, es considerar que las modificaciones neurobiológicas inducidas por el estrés en los hombres son adaptativas, lo que posiblemente vendría a prevenir la aparición de estos trastornos (Altemus, 2009). Es decir, aunque los estudios indican que las mujeres están protegidas de los efectos neurobiológicos y comportamentales del estrés, estas modificaciones que sí se observan en los hombres, tendrían en éstos un carácter adaptativo a largo plazo protegiéndoles de desarrollar trastornos de ansiedad.

Se considera que son los estrógenos, con sus efectos neuroprotectores, los que contribuyen a la resistencia de las mujeres al estrés. Estas hormonas modulan, en función de la dosis administrada, la actividad de los dos sistemas principales de respuesta al estrés, el eje HPA y el sistema nervioso autónomo (Altemus, 2009; Horst et al., 2009), llegando incluso a suprimir su respuesta tanto en las mujeres (Roca et al., 2003) como en los roedores (Young et al., 2001). Pero los andrógenos y la progesterona modifican de la misma forma la respuesta del eje HPA al estrés, por lo que también contribuirán a las diferencias sexuales observadas.

Así, para poder entender las posibles causas de las diferencias sexuales en la respuesta al estrés, hay que profundizar en la relación entre el eje Hipotalámico-Pituitario-Gonadal (HPG) y el eje HPA. Los esteroides gonadales pueden influir en los mecanismos de regulación del eje HPA a través de sus efectos sobre los receptores de los glucocorticoides, sobre los sistemas cerebrales de la CRH, y sobre la respuesta de la hipófisis y la respuesta adrenal a la CRH (Young et al., 2008). Se ha observado que las ratas hembras en la fase proestro, cuando los estrógenos presentan sus niveles más altos, incrementaban su respuesta al estrés en comparación a las hembras en otras fases del ciclo estral, incremento que era abolido con la ovariectomia y que se conseguía reinstaurar con la administración exógena de estradiol (Paris et al., in press; Young et al., 2008). De hecho, se ha comprobado que el estradiol estimula directamente la expresión de ARNm de la CRH en el núcleo paraventricular del hipotálamo, provocando un incremento en la liberación de ACTH/corticosterona en respuesta a un estresor mayor en las ratas hembras en la fase proestro que en las ratas machos (Iwasaki-Sekino et al., 2009). Sin embargo, las hembras ovariectomizadas seguían mostrando una mayor hiperreactividad al estrés que los machos intactos, posiblemente debido a un efecto inhibitorio de la testosterona sobre la respuesta de estrés (Paris et al., in press ; Young et al., 2008).

Contrariamente en las mujeres, se conoce que los niveles de cortisol en saliva cambian a lo largo del ciclo menstrual, encontrando sus niveles mayores en los días 1-5 del ciclo, cuando los niveles de estrógenos y progesterona están más bajos. Además, la elevación de los niveles de progesterona se ha asociado con un incremento de la respuesta de cortisol ante un estresor físico agudo (Paris et al., in press). Desafortunadamente, son escasos los estudios en seres humanos que controlan la fase del ciclo menstrual en la que se encuentran las mujeres investigadas.

Algunos trabajos han destacado el papel de la oxitocina y la vasopresina en las diferencias sexuales comportamentales observadas en la respuesta al estrés. La oxitocina reduce el temor, el dolor y la respuesta de estrés, potenciando al mismo tiempo los comportamientos de crianza, sexual y social. De hecho, se ha comprobado en mujeres que las respuestas al estrés están reducidas durante el embarazo y la lactancia, cuando las concentraciones de esta hormona son muy elevadas. Con respecto a la vasopresina, se han observado diferencias sexuales en las neuronas productoras de esta hormona, localizadas en los núcleos supraóptico y paraventricular del hipotálamo, siendo más grandes y mostrando una mayor actividad neuronal en hombres que en mujeres. Esta observación es congruente con el hallazgo de que la vasopresina puede provocar una respuesta del eje HPA al estrés mayor en los hombres que en las mujeres (Altemus, 2009).

Revisando la literatura existente sobre la relación entre los esteroides gonadales y el eje HPA, está claro que estas hormonas tienen un papel modulador en la regulación del eje, tanto en animales experimentales como en seres humanos. Aunque los mecanismos precisos todavía permanecen sin aclarar totalmente, es muy probable que tanto los estrógenos como las 
progestinas actúen sobre múltiples niveles del eje HPA. La evidencia experimental apoya un efecto antiglucocorticoide de la progesterona, mediado en parte por su acción sobre los receptores de los glucocorticoides. También, es probable que el estradiol tenga un papel central sobre la mayor resistencia de las hembras a inhibir el feedback del eje HPA. Estudios en roedores han demostrado que el tratamiento con estradiol exógeno incrementa o disminuye la respuesta de estrés dependiendo de la dosis y/o el periodo de exposición a los estrógenos (Solomon y Herman, 2009). Además, hay que tener presente que el estradiol puede incrementar la sensibilidad de la glándula adrenal al ACTH circulante, ofreciendo así un mecanismo periférico adicional para la hipersecreción de glucocorticoides en las hembras (Young et al., 2008).

Es posible que estas interacciones entre los esteroides gonadales y el eje HPA contribuyan a incrementar la prevalencia de los trastornos de ansiedad en las mujeres en comparación con los hombres (Young et al., 2008), pero es evidente que hacen falta todavía muchos más estudios para poder entender qué papel juega el sexo en las psicopatologías.

\section{Conclusión}

A lo largo de la historia, la medicina y la psiquiatría han utilizado prioritariamente machos en la investigación científica (Hughes, 2007). De manera que gran parte de los estudios sobre la etiología, curso o tratamiento de las enfermedades mentales se han realizado en sujetos del sexo masculino y no se ha reconocido que, posiblemente, existían importantes diferencias de sexo. De hecho, aunque hemos visto que los trastornos de ansiedad aparecen con mucha mayor frecuencia en mujeres, la mayoría de estudios con animales experimentales sobre estas patologías han sido llevados a cabo sólo con machos, incluso aunque las respuestas de miedo en roedores son análogas a las reacciones de ansiedad en seres humanos en términos de sustratos biológicos y ambientales (Hughes, 2007; Palanza, 2001). Este asunto no ha recibido, ni recibe la atención necesaria y resulta sorprendente la escasez de estudios realizados con el sexo femenino a lo largo de la historia de las neurociencias. Las razones de esta discriminación son inexplicables y la mayoría erróneas. En la investigación básica, se prefiere el empleo de sujetos del sexo masculino principalmente porque se considera que las fluctuaciones hormonales del ciclo estral en las hembras provocan, sobre todo en las medidas conductuales, una mayor variabilidad entre sujetos. Esta creencia ha sido desmentida en numerosos estudios, encontrándose una variabilidad intersujeto similar entre los sexos o incluso en ocasiones mayor en los machos (Hughes, 2007; Parra et al., 1999). Otra razón errónea para defender la utilización sólo de machos, es argumentar que no existen diferencias de sexo en las medidas estudiadas, por lo que no es necesario utilizar sujetos de ambos sexos resultando menos costosa así la investigación. Pero resulta curioso que cuando los estudios utilizan sólo sujetos del sexo masculino, no es justificado por los autores la utilización de un solo sexo, mientras que utilizar sólo hembras o ambos sexos, es justificado por los investigadores en los artículos (Hughes, 2007).

Como hemos expuesto, realmente no se conoce por qué las tasas de ansiedad son tan elevadas en las mujeres y se sabe muy poco sobre cuales son los factores de riesgo antecedentes. Se especula que pueden existir factores de vulnerabilidad (bioquímicos, hormonales, sociales) que expliquen esta preponderancia a los trastornos de ansiedad en la mujer. Sin embargo, hasta la fecha, no se han identificado ni dilucidado los factores que originan las diferencias de sexo en la etiología y patofisiología de los trastornos de ansiedad, siendo necesaria más investigación sobre el tema.

\section{Referencias}

Alonso, J., Angermeyer, M.C., Bernert, S., Bruffaerts, R., Brugha, T.S., Bryson, H., Girolamo, R., Graaf, K., Demyttenaere, I., Gasquet, J.M., Haro, S.J., Katz, R.C., Kessler, V., Kovess, J.P., Lépine, J., Ormel, G., Polidori, L.J., Russo, G., Vilagut, J., Almansa, S., Arbabzadeh-Bouchez, J., Autonell, M., Bernal, M.A., Buist-Bouwman, M., Codony, A., Domingo-Salvany, M., Ferrer, S.S., Joo, M., MartínezAlonso, H., Matschinger, F., Mazzi, Z., Morgan, P., Morosini, C., Palacín, B., Romera, N., Taub, W.A. y Vollebergh, W.A.M. (2004). Prevalence of mental disorders in Europe: Results from the European Study of the Epidemiology of Mental Disorders (ESEMeD) project. Acta Psychiatrica Scandinavica, 109 (Suppl. 420), 21-27.

Altemus, M. (2009). Neurobiología, sexo y género. En W.E. Narrow, M.B. First, P.J. Sirovatka y D.A. Regier (Eds.). Agenda de investigación para el DSM-V. Consideraciones sobre la edad y el género en el diagnóstico psiquiátrico, (pp. 47-62) Barcelona: Elsevier Masson.

Altemus, M. y Epstein, L. (2008). Sex differences in anxiety disorders. En J.B. Becker, K.J. Berkley, N. Geary, E. Hampson, J.P. Herman y E.A. Young. Sex differences in the brain, (pp. 397-404) Oxford University Press.

American Psychiatric Association (2000). Diagnostic and statistical manual of mental disorders: DSM-IV-TR. Washington, DC : American Psychiatric Association.

Angst J. (1997). Epidemiology of social phobia. European Neuropsychopharmacology, 7, S84-S85.

Asami, T., Yamasue, H., Hayano, F., Nakamura, M., Uehara, K., Otsuka, T., Roppongi, T., Nihashi, N., Inoue, T. y Hirayasu, Y. (2009). Sexually dimorphic gray matter volumen reduction in patients with panic disorder. Psychiatry Research: Neuroimaging, 173, 128-134.

Bogetto, F., Venturello, S., Albert, U., Maina, G., Gand, L. y Ravizza, A. (1999). Gender-related clinical differences in obsessive-compulsive disorder. European Psychiatry, 14, 434-441.

Cahill, L., Haier, R.J., White, N.S., Fallon, J., Kilpatrick, L., 
Lawrence, C., Potkin, S.G., Alkire, M.T. (2001). Sexrelated difference in amygdale activity during emotionally influenced memory storage. Neurobiology of Learning and Memory, 75, 1-9.

Cahill, L. (2003). Sex- and hemisphere-related influences on the neurobiology of emotionally influenced memory. Progress in Neuropsychopharmacology and Biological Psychiatry, 27, 1235-1241.

Cahill, L. (2006). Why sex matter for neuroscience. Nature Reviews Neuroscience, 7, 477-484.

Cahill, L. (2009). Dimorfismo sexual cerebral. Temas 56: Instinto sexual. Investigación y Ciencia, 56, 62-70.

Carrasco-Galán, I. y Espinar-Fellmann, I. (2008). Trastornos de ansiedad y género. Mente y Cerebro, 31, 12-21.

Chua, P., y Dolan, J. (2000). The neurobiology of anxiety and anxiety-related disorders: A functional neuroimaging perspective. En: J.C. Mazziotta y A.W. Toga (Eds.). Brain Mapping: The Disorders, (pp. 509-522). San Diego, CA: Academic Press.

Dalla, C. y Shors, T.J. (2009). Sex differences in learning processes of classical and operant conditioning. Physiology and Behavior, 97, 229-238.

Darnall, B.D. y Suarez, E.C. (2009). Sex and gender in psychoneuroimmunology research: Past, present and future. Brain, Behavior, and Immunity, 23, 595-604.

Eaton, W., Kessler, R.C., Wittchen, H.U. y Magee, W.J. (1994). Panic and panic disorder in the United States. American Journal of Psychiatry, 151, 413-420.

Grant, B.F. y Weissman, M.M. (2009). Género y prevalencia de los trastornos psiquiátricos. En: W.E. Narrow, M.B. First, P.J. Sirovatka y D.A. Regier (Eds.). Agenda de investigación para el DSM-V. Consideraciones sobre la edad y el género en el diagnóstico psiquiátrico, (pp. 31-45) Barcelona: Elsevier Masson.

Horst, G.J., Wichmann, R., Gerrits, M., Westenbroek, C. y Lin, Y. (2009). Sex differences in stress responses: Focus on ovarian hormones. Physiology and Behavior, 97, 239-249.

Hughes, R.N. (2007). Sex does matter: comments on the prevalence of male-only investigations of drug effects on rodent behaviour. Behavioural Pharmacology, 18, 583-589.

Iwasaki-Sekino, A., Mano-Otagiri, A., Ohata, H., Yamauchi, N. y Shibasaki, T. (2009). Gender differences in corticotropin and corticosterone secretion and corticotropin-releasing factor mRNA expression in the paraventricular nucleus of the hypothalamus and the central nucleus of the amygdala in response to footshock stress or psychological stress in rats. Psychoneuroendocrinology, 34, 226-237.

Kendler, K.S., Myers, J. y Prescott, C.A. (2005). Sex differences in the relationship between social support and risk for major depression: a longitudinal study of opposite-sex twin pairs. American Journal of Psychiatry, 162, 250-256.

Kessler, R., Sonnega, A., Bromet, E., Hughes, M. y Nelson, C. (1995). Post-traumatic stress disorder in the National
Comorbidly Survey. Archives of General Psychiatry, 52, 1048-1060.

Kessler, R.C., McGonagle, K.A., Zhao, S., Nelson, C., Hughes, M., Eshleman, S., Wittchen, H.U. y Kendler, K.S. (1994). Lifetime and 12 month prevalence of DSM-III-R psychiatric disorders in the United States: results from the national comorbidity survey. Archives of General Psychiatry, 51, 8-19.

Kim, I.R., Min, S.K. y Yu, B.E. (2004). Differences in betaadrenergic receptor sensibility between women and men with panic disorder. European Neuropsychopharmacology, 14, 515-520.

Kudielka, B,M., Buske-Kirschbaum, A., Hellhanmer, D.H. y Kirschbaum, C. (2004). HPA axis responses to laboratory psychosocial stress in healthy elderly adults, younger adults, and children: impact of age and gender. Psychoneuroendocrinology, 29, 83-98.

Kudielka, B.M. y Kirschbaum, C. (2005). Sex differences in HPA axis responses to stress: a review. Biological Psychology, 69, 113-132.

Le Melledo, J.M., Van Driel, M., Coupland, N.J., Lott, P. y Jhangri, G.S. (2000). Response to flumazenil in women with premenstrual dysphoric disorder. American Journal of Psychiatry, 157, 821-823.

Leskin, G.A. y Sheikh, J.I. (2004). Gender differences in panic disorder, Psychiatric Times, 21, 1-6.

Lochner, C., Hemmings, S.M., Kinnear, C.J., Moolman-Smook, J.C., Corfield, V.A., Knowles, J.A., Niehaus, D.J. y Stein, D.J. (2004). Gender in obsessive-compulsive disorder: clinical and genetic findings. European Neuropsychopharmacology, 14, 105-113.

McLean, C.P. y Anderson, E.R. (2009). Brave men and timid women? A review of the gender differences in fear and anxiety. Clinical Psychology Review, 29, 496-505.

McEwen, B.S. y Alves, S.E. (1999). Estrogen actions in the central nervous system. Endocrine Reviews, 20, 279-307.

McEwen, B.S. (2002). The neurobiology and neuroendocrinology of stress: Implications for post-traumatic stress disorder from a basic science perspective. Psychiatric Clinics of North America, 25, 469-494.

McEwen, B.S. (2007). Physiology and neurobiology of stress and adaptation:central role of the brain. Physiological reviews, 87, 873-904.

Olff, M., Langeland, W., Draijer, N. y Gersons, B.P. (2007). Gender differences in posttraumatic stress disorder. Psychological Bulletin, 133, 183-204.

OMS. (2000). Women's mental health: An evidence based review. Informe sobre la salud en el mundo.

OMS. (2001). Salud mental: nuevos conocimientos, nuevas esperanzas. Informe sobre la salud en el mundo.

Palanza, P. (2001). Animal models of anxiety and depresión: how are females different? Neuroscience and Biobehavioral Reviews, 25, 219-233. 
Paris, J.J, Franco, C., Sodano, R., Freidenberg, B., Gordis, E., Anderson, D.A., Forsyth, J.P., Wulfert, E. y Frye, C.A. (2009). Sex differences in salivary cortisol in response to acute stressors among healthy participants, in recreacional or pathological gambers, and in those with posttraumatic stress disorder. Hormones and Behavior, in press.

Parra, A., Arenas, M.C., Monleón, S., Vinader-Caerols, C. y Simón, V.M. (1999). Sex differences in the effects of neuroleptics on escape-avoidance behavior in mice: A review. Pharmacology Biochemistry and Behavior, 64, 813-820.

Peer, M., Soares, C. y Steiner, M. (2008). The complex interrelationships of menstrual cyclicity and anxiety disorders. Psychiatric Times, 25, 1-14.

Phillips, K.A. y First, M.B. (2009). Introducción. En W.E. Narrow, M.B. First, P.J. Sirovatka y D.A. Regier (Eds.). Agenda de investigación para el DSM-V. Consideraciones sobre la edad y el género en el diagnóstico psiquiátrico (pp. 3-6) Barcelona: Elsevier Masson.

Pooley, E.C., Fineberg, N. y Harrison, P.J. (2007). The met(158) allele of catechol-O-methyltransferase (COMT) is associated with obsessive-compulsive disorder in men: case-control study and meta-analysis. Molecular Psychiatry, 12, 556-561.

Roca, C.A., Schmidt, P.J., Altemus, M., Deuster, P., Danaceau, M.A., Putnam, K. y Rubinow, D.R. (2003). Diferential menstrual cycle regulation of hypothalamic-pituitary-adrenal axis in women with premenstrual syndrome and controls. The Journal of Clinical and Endocrinology Metabolism, 88, 3057-3063.

Seeman, M.V. (1997). Psychopathology in women and men: focus on female hormones. American Journal of Psychiatry, 154, 1641-1647.

Solomon, M.B. y Herman, J.P. (2009). Sex differences in psychopathology: Of gonads, adrenals and mental illness. Physiology and Behavior, 97, 250-258.

Taylor, S.E., Klein, L.C., Lewis, B.P., Gruenewald, T.L., Gurung, R.A.R. y Updegraff, J.A. (2000). Biohevavioral responses to stress in females: Tend-and-befriend, not fightor-flight. Psychological Review, 107, 411-429.

Tolin, D.F. y Foa, E.B. (2006). Sex differences in trauma and posttraumatic stress disorder: a quantitative review of 25 years of research. Psychological Bulletin, 132, 959-992.

Torresan R.C., Ramos-Cerqueira A.T., De Mathis M.A., Diniz J-B-, Ferrão Y.A., Miguel, E.C. y Torres, A.R. (2009). Sex differences in the phenotypic expression of obsessive-compulsive disorder: an exploratory study from Brazil. Comprenhsive Psychiatry, 50, 63-69.

Wang, A.Y. Wang, J.F. Samuels, Y.C. Chang, M.A., Grados, M.A., Greenberg, B.D., Knowles, J.A., McCracken, J.T., Rauch, S.L., Murphy, D.L., Rasmussen, S.A., Cullen, B., Hoehn-Saric, R., Pinto, A., Fyer, A.J., Piacentini, J., Pauls, D.L., Bienvenu, O.J., Riddle, M., Shugart, Y.Y., Liang, K.Y. y Nestadt, G. (2009). Gender differences in genetic linkage and association on $11 \mathrm{p} 15$ in obsessive-compulsive disorder families. American Journal of Medical Genetics, 150, 33-40.

Weissman, M.M., Bland, R.C., Canino, G.J., Greenwald, S., Hwu, H.G., Lee, C.K., Newman, S.C., Oakley-Browne, M.A., Rubio-Stipec, M., Wickramaratne, P.J., et al (1994). The cross national epidemiology of obsessive compulsive disorder. Journal of Clinical Psychiatry, 55, 5-10.

Wilson, S.N., Van Der Kolk, B., Burbridge, J., Fisler, R. y Kradin, R. (1999). Phenotype of blood lymphocytes in PTSD suggests chronic immune activation. Psychosomatics, 40, 222-225.

Wisner, K.L. y Dolan-Sewell, R. (2009). ¿Por qué tiene importancia el género? En: W.E. Narrow, M.B. First, P.J. Sirovatka y D.A. Regier (Eds.). Agenda de investigación para el DSM-V. Consideraciones sobre la edad y el género en el diagnóstico psiquiátrico (pp. 7-17). Barcelona: Elsevier Masson.

Yehuda, R. y LeDoux J. (2007). Response variation following trauma: A translational neuroscience approach to understanding PTSD. Neuron, 56, 19-32.

Yonkers, K.A. y Kidner, C.L. (2002). Sex differences in anxiety disorders. En F. Lewis-Hall, T.S. Williams, J.A. Panetta y J.M. Herrera (Eds.). Psychiatric illness in women (pp. 5-30). American Psychiatric Publishing.

Young, E.A. (1998). Sex differences and the HPA axis: implications for psychiatric disease. The Journal of Gender-Specific Medicine, 1, 21-27.

Young, E.A., Altemus, M., Parkinson, V. y Shastry, S. (2001). Effect of estrogen antagonists and agonists on the ACTH response to restraint stress in female rats. Neuropsychopharmacology, 25, 881-891.

Young, E.A., Korszun, A., Figueiredo, H.F., Banks-Solomon, M. y Herman, J.P. (2008). Sex differences in HPA Axis regulation. En J.B. Becker, K.J. Berkley, N. Geary, E. Hampson, J.P. Herman y E.A. Young. Sex differences in the brain: from genes to behaviour (pp. 95-105). Oxford University Press.

Fecha de recepción: 8 de octubre de 2009 Fecha de aceptación: 24 de noviembre de 2009 